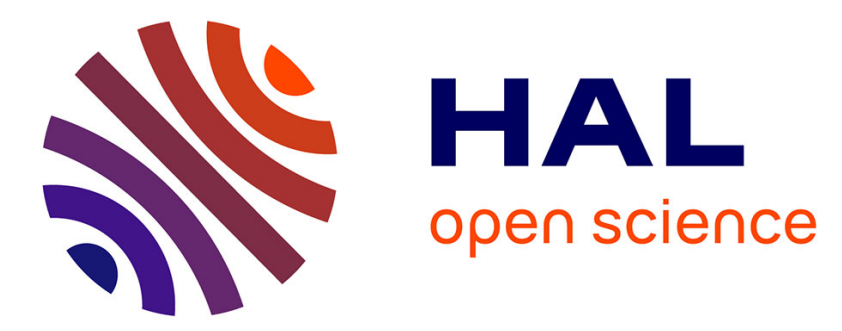

\title{
Helium-vacancy interactions in vanadium and tantalum
}

Wesley Unn-Toc, Mihai-Cosmin Marinica, Thomas Jourdan

\section{To cite this version:}

Wesley Unn-Toc, Mihai-Cosmin Marinica, Thomas Jourdan. Helium-vacancy interactions in vanadium and tantalum. Computational Materials Science, 2018, 145, pp.197-207. 10.1016/j.commatsci.2017.12.011 . cea-02428738

\section{HAL Id: cea-02428738 https://hal-cea.archives-ouvertes.fr/cea-02428738}

Submitted on 6 Jan 2020

HAL is a multi-disciplinary open access archive for the deposit and dissemination of scientific research documents, whether they are published or not. The documents may come from teaching and research institutions in France or abroad, or from public or private research centers.
L'archive ouverte pluridisciplinaire HAL, est destinée au dépôt et à la diffusion de documents scientifiques de niveau recherche, publiés ou non, émanant des établissements d'enseignement et de recherche français ou étrangers, des laboratoires publics ou privés. 


\title{
Helium-vacancy interactions in vanadium and tantalum
}

\author{
Wesley Unn-Toc, Mihai-Cosmin Marinica*, Thomas Jourdan \\ DEN-Service de Recherches de Métallurgie Physique, CEA, Université Paris-Saclay, F-91191 Gif-sur-Yvette, France
}

\section{A R T I C L E I N F O}

\section{Article history:}

Received 11 August 2017

Received in revised form 5 December 2017

Accepted 6 December 2017

Available online 9 January 2018

\section{Keywords:}

Density Functional Theory

He-vacancy

Defects

Energy landscape

Fusion

Tantalum

Vanadium

\begin{abstract}
A B S T R A C T
In this paper we investigate the energy landscape of vacancy clusters in the presence of Helium in two materials, Vanadium and Tantalum. In order to address the relative stability of small vacancy-Helium clusters as well as the basic migration mechanisms we have used Density Functional Theory. The results of calculations are compared to experimental results, when available, and can be systematized in potentially simple laws that can be easily used subsequently in multi-scale techniques including kinetic Monte Carlo and cluster dynamics simulations.
\end{abstract}

(ㄷ) 2017 Elsevier B.V. All rights reserved.

\section{Introduction}

The main materials of interest for fission and fusion application are the body centered cubic (bcc) metals [1,2]. Irradiation of bcc transition metals leads to a large number of point defects in the form of self-interstitial atoms and vacancies. The interactions and migrations of defects as well as their absorption at sinks, like dislocations and grain boundaries, can influence microstructural transformations in material which can affect various properties of the metals. Experimental and theoretical studies [3] have shown that the body centered cubic lattice demonstrates improved radiation resistance compared to the close-packed face centered cubic lattice due to reduced amount of vacancy and interstitial defect clustering that occurs directly within displacement cascades as well as higher stacking fault energy. In this paper we will focus on the vacancy clusters and their interactions with Helium atoms in two materials which are good candidates for future fusion systems, $\mathrm{V}$ and Ta [4].

$\mathrm{V}$ and its alloys are promising candidates as blanket for fusion reactor thanks to their mechanical properties at high temperature and low activation [5,6]. V alloys demonstrate a good combination of strength, ductility and radiation resistance [4,5]. Ta is known for its easy manufacturing, high toughness, high-sputtering threshold energy, and low activation properties which make it a candidate for target material for spallation sources or even first wall material

\footnotetext{
* Corresponding author.

E-mail address: mihai-cosmin.marinica@cea.fr (M.-C. Marinica).
}

in fusion reactors $[7,8]$. As such, study of defects in those two materials, V and Ta serve as the basic foundation for future research regarding structural materials in the fusion industry.

Under irradiation, the structural materials are subject to damages such as swelling [9], embrittlement [10] and blistering [11] triggered, enhanced or suppressed [12] by He resulting of $(n, \alpha)$ transmutation reactions. He atoms are usually trapped at grain boundaries, dislocations or voids $[13,14]$. The early stages of formation of He bubbles in metals cannot be observed experimentally due to the limited spatial resolution. Ab initio calculations based on Density Functional Theory (DFT) are powerful tools to investigate the processes taking place at the beginning of nucleation of such objects. DFT calculations provide quantitative insight into the nature of clusters containing a small number of defects. About $\mathrm{V}$ relevant studies can only be found in the last years while data concerning Ta remains scarce. Hua et al. [15] went to the conclusion that alloying $\mathrm{V}$ with $\mathrm{Ti}$ enhances migration energy of He and diminishes He-vacancy binding energy. Zhang et al. [16] studied the interaction of He with monovacancy and SIA in pure $\mathrm{V}$ and $\mathrm{V}$ alloys. They showed that in $\mathrm{V}$ alloys, the formation and migration energies of He are larger than in pure $V$. The same group investigated stability of small He-vacancy clusters and migration of monovacancy. It was found that interstitial He clusters are weakly bound and need vacancy to be stable [17]. The formation of He and vacancy at grain boundary in V has been investigated in Ref. [18]. $\mathrm{He}-\mathrm{He}$ and He-metal interactions have been studied in various bcc transition metals [19]. Energetics of interstitial He in several metals of $V$ group and VI group have been detailed in Ref. [20]. Up to 
now, however, only a limited number of configurations has been studied. In this study we focus on He-vacancy type clusters in pure $\mathrm{V}$ and $\mathrm{Ta}$, in particular we are interested in the first steps of nascent bubbles. To this end, we detail the formation and migration not only of He and monovacancy but also di-vacancies that have been proved to play a significant role in diffusion processes in Fe [21]. In the above-mentioned works, a small amount of clusters have been studied. Attempting to get the most stable clusters, we explore systematically several configurations of each He-vacancy cluster type.

This paper is organized as follows: Section 2 provides the methodology and the details of the employed techniques used for the calculations. In Section 3.1 we present the formation energies computed for vacancy and He-vacancy type clusters. In Section 3.2 the stability of He-vacancy type clusters is investigated. Finally, Section 3.3 describes the migration processes and barriers of vacancy clusters and how helium modifies the diffusion mechanisms.

\section{Computational details}

$A b$ initio calculations were performed with density functional theory (DFT) as implemented in the Vienna $A b$ initio Simulation Package (VASP) [22,23]. We employed non spin-polarised calculations with the generalised gradient approximation (GGA) with the Perdew-Burke-Ernzerhof (PBE) [24] exchange-correlation functional, and semi-core projector-augmented wave (PAW) $[25,26]$ as pseudo-potentials. According to Ref. [19], Van der Waals interactions $(\sim 20 \mathrm{meV})$ can be neglected with respect to He-He and He-metal interactions. Up to 3 vacancies, the bcc supercell of 128 atoms with a $4 \times 4 \times 4 k$-points mesh grid generated by Monkhorst-Pack scheme [27] was used; for more than 3 vacancies we have used a 250 atoms supercell with a $3 \times 3 \times 3 k$-points mesh grid. The Methfessel-Paxton smearing [28] with a $0.25 \mathrm{eV}$ width was used and a $350 \mathrm{eV}$ cutoff energy for both $\mathrm{V}$ and Ta case. Atomic positions were relaxed at constant volume until forces on each atom were less than $0.01 \mathrm{eV} / \AA$. The migration barriers and paths were determined using the climbing image nudged elastic band method (CI-NEB) $[29,30]$ with $5 \mathrm{eV} / \AA^{2}$ spring constant and a $0.01 \mathrm{eV} / \AA$ force convergence threshold. In addition, the converged energy it was corrected by subtracting the elastic dipole interaction energy between self-images due to periodic boundary conditions. We applied the procedure from [31]. In that paper, the elastic dipole-dipole correction is computed from the value of elastic dipole of the defects, which, in first approximation, is proportional to the stress of the simulation box. In order to have reliable value of the elastic dipole, from the stress of the simulation box containing the defect we have extracted the residual stress of the bulk reference. The magnitude of corrections are always smaller than $0.15 \mathrm{eV}$.

The equilibrium bcc lattice constant obtained for $\mathrm{V}$ and $\mathrm{Ta}$ was $3.00 \AA$ and $3.32 \AA$, respectively. Those values are close to experimental data $3.03 \AA$ for $\mathrm{V}$ and $3.30 \AA$ for Ta [32].

\section{Results}

\subsection{Formation energy of $\mathrm{He}_{n} V_{m}$ clusters}

The energetics of isolated He in interstitial configurations as well as the vacancy and vacancy-helium clusters inserted in the bcc matrix of $\mathrm{V}$ and $\mathrm{Ta}$ are investigated.

The formation energy of the simulation box containing $n \mathrm{He}$ atoms inserted into the bcc matrix is given by:

$E_{\mathrm{He}_{n}}^{f}=E_{\mathrm{He}_{n}}-E_{0}-n \times E_{\mathrm{He}}$,
$E_{\mathrm{He}_{n}}$ is the DFT energy of the supercell containing $n$ interstitials He, $E_{0}$ is the energy of the perfect bulk supercell and, finally, $E_{\mathrm{He}}$ is the energy of an isolated He atom. $E_{\mathrm{He}}(0.014 \mathrm{eV})$ has been computed with single He atom in a $10 \AA \times 10 \AA \times 10 \AA$ box, a $400 \mathrm{eV}$ cutoff energy and a pseudo-potential with $1 s^{2}$ electronic structure. Firstly, we carefully checked the case $n=1$ of only one He atom in the bcc matrix. Lately, we will discuss the case $n>1$. In $\mathrm{V}$ the formation energies for interstitial configurations are $3.12 \mathrm{eV}$ and $3.34 \mathrm{eV}$ for tetrahedral $(T)$ and octahedral sites $(O)$, respectively. In Ta the same relative stability is found, the interstitial tetrahedral configuration having a formation energy of $3.47 \mathrm{eV}$, with $0.32 \mathrm{eV}$ lower than interstitial octahedral configuration. For both metals, $\mathrm{V}$ and $\mathrm{Ta}$, we found the similar hierarchy in the energy of interstitial configurations, the $T$ interstitial site is more stable than the $O$ site, as in other bcc transition metals, such as Fe [33], W and Nb [20].

In both metals, $\mathrm{V}$ and $\mathrm{Ta}$, we have studied also the relative stability of vacancy clusters $\left(V_{m}\right)$ up to quadri-vacancy $(m=4)$. Again we have used as indicator the zero $\mathrm{K}$ formation energy of clusters. The formation energy of the $m$ vacancy cluster, $V_{m}$, is defined by:

$E_{V_{m}}^{f}=E_{V_{m}}-\frac{(N-m)}{N} \times E_{0}$,

with $N$ the number of atoms of the perfect bcc lattice and $E_{V_{m}}$ the DFT energy of the supercell containing $m$ vacancies agglomerated in a cluster. Among many investigated configurations the lowest energy cluster, for the cases $m=2,3,4$, is found to be the more compact clusters, which are reported in Fig. 1. The formation energies of mono-vacancy (Table 1) are in good agreement with experiments for both $\mathrm{V}(2.47 \mathrm{eV})[34,35]$ and $\mathrm{Ta}(2.88 \mathrm{eV})[34,36,37]$. We point here the exception of the di-vacancy cluster. In Ta and V we find that the energy of 2nd nearest neighbour configuration (hereafter noted $V_{2}^{2 n n}$ ) is lower than the energy of 1 st nearest neighbour configuration (hereafter denoted $V_{2}^{1 n n}$ ). The formation energy of $V_{2}^{2 n n}$ is lower than the formation energy of the configuration $V_{2}^{1 n n}$ by 0.19 $\mathrm{eV}$ and $0.24 \mathrm{eV}$ in $\mathrm{V}$ and Ta, respectively (Table 1 ). The same tendency is observed for all VIB group and VB group metals and iron for which the most stable configuration of di-vacancy is the $V_{2}^{2 n n}$. In $\mathrm{V}$ and $\mathrm{Ta}$, the binding energy of di-vacancy, in both configurations, $V_{2}^{1 n n}$ and $V_{2}^{2 n n}$, is positive, indicating the attraction between mono-vacancies in order to form the di-vacancy. The same tendency for the di-vacancy energy landscape is observed in iron and

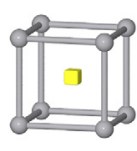

(a)

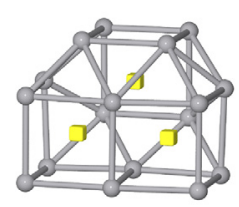

(d)

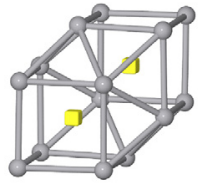

(b)

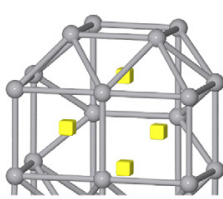

(e) (c)

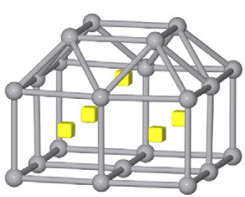

(f)

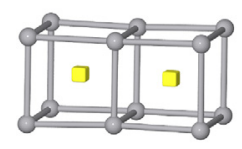

Fig. 1. The configurations of vacancy clusters $\left(V_{m}, m=1,2,3,4,5\right)$ with the lowest formation energy. Vacancies are represented by cubes while the atoms of the bcc lattice are represented with spheres. For the case of di-vacancy we show the first nearest neighbour $\left(V_{2}^{1 n n}\right)$ and the second nearest neighbour configuration $V_{2}^{2 n n}:$ (a) $V_{1}$, (b) $V_{2}^{1 n n}$, (c) $V_{2}^{2 n n}$, (d) $V_{3}$, (e) $V_{4}$, (f) $V_{5}$. 
Table 1

The lower limit of formation energy (in eV) of $\mathrm{He}_{n} V_{m}$ clusters, extracted from the present calculations, using Eq. (3), for small values of $n$ and $m$.

\begin{tabular}{|c|c|c|c|c|c|c|c|c|c|c|c|c|}
\hline \multirow{2}{*}{$\mathrm{n}$} & \multicolumn{6}{|c|}{ V } & \multicolumn{6}{|c|}{ Ta } \\
\hline & 0 & 1 & $2(1 \mathrm{nn})$ & $2(2 n n)$ & 3 & 4 & 0 & 1 & $2(1 \mathrm{nn})$ & $2(2 n n)$ & 3 & 4 \\
\hline 0 & & $\begin{array}{c}2.47 \\
(2.1 \pm 0.2)^{\mathrm{a}} \\
(2.2 \pm 0.4)^{\mathrm{b}}\end{array}$ & 4.69 & 4.50 & 6.46 & 8.07 & & $\begin{array}{c}2.88 \\
(2.8 \pm 0.6)^{\mathrm{a}} \\
(3.1)^{\mathrm{c}} \\
(2.9 \pm 0.4)^{\mathrm{d}}\end{array}$ & 5.66 & 5.42 & 7.97 & 9.96 \\
\hline 1 & 3.12 & 4.47 & 6.15 & 6.06 & 7.58 & 8.88 & 3.47 & 4.66 & 6.89 & 6.92 & 8.77 & 10.44 \\
\hline 2 & 6.16 & 6.26 & 7.54 & 7.94 & 8.90 & 9.81 & 6.84 & 6.49 & 8.22 & 8.40 & 9.97 & 11.31 \\
\hline 3 & 9.15 & 8.15 & 9.18 & 9.74 & 10.09 & 10.84 & 10.00 & 8.63 & 9.69 & 10.07 & 11.09 & 12.28 \\
\hline 4 & 12.03 & 10.01 & 10.92 & 11.48 & 11.74 & 11.92 & 13.26 & 10.79 & 11.22 & 11.80 & 12.29 & 13.31 \\
\hline 5 & & 11.91 & 12.47 & 13.45 & 12.99 & 13.20 & & & 12.84 & 14.19 & 13.81 & 14.46 \\
\hline 6 & & 13.73 & 14.44 & 15.07 & 14.62 & 14.76 & & 14.87 & 14.80 & 15.69 & 15.39 & 15.62 \\
\hline
\end{tabular}

a Experimental data from Ref. [34].

b Experimental data from Ref. [35].

c Experimental data from Ref. [36].

d Experimental data from Ref. [37].

is at variance with VIB metals, such as Mo and W [38-43], for which the first nearest neighbour configuration is slightly attractive while the second nearest neighbour configuration is strongly repulsive.

The formation energy for mixed He-vacancy clusters, $\mathrm{He}_{n} V_{m}$ (up to $n=26$ for $m=4$ ), is defined as:

$E_{\mathrm{He}_{n} V_{m}}^{f}=E_{\mathrm{He}_{n} V_{m}}-\frac{(N-m)}{N} \times E_{0}-n \times E_{\mathrm{He}}$,

where $\mathrm{E}_{\mathrm{He}_{n} V_{m}}$ is the DFT energy of the supercell containing the $\mathrm{He}_{n} V_{m}$ cluster. The morphology and construction of all possible clusters for a given number of $n$ He atoms and $m$ vacancies is not simple task. The number of possible choices grows exponentially with the number of defects. The exhaustive search is difficult and consequently several hypotheses are made in order to choose the geometries of the lowest energy configurations. Firstly, we investigated only geometrically compact configurations. The only exception was the di-vacancy for which the two configurations first and second nearest neighbours have been chosen. Secondly, for any given $\mathrm{He}_{n} V_{m}$ cluster the $n$ He atoms randomly decorate the most stable $V_{m}$ clusters, deduced previously and presented in Fig. 1. Therefore, for a cluster $\mathrm{He}_{n} V_{m}$ tens of random positions have been picked for the $n$ He atoms. These atoms are placed in $T, O$ or substitutional $(S)$ sites in the neighborhood of the $m$ vacancies. For Ta, we reduce the sampling up to ten configurations, as we will see later, He atoms prefer to occupy $S$ sites and many initial configurations collapse to the same minimum. Nevertheless, since we restrict the sampling, there is no guarantee that we will find the most stable clusters. This is critical in particular for the largest clusters, which are beyond the scope of this paper. However, for the smallest clusters, which are the object of the present paper, we are reasonably confident in finding the lowest energy configurations.

In $\mathrm{V}$, the lowest energy mixed configuration $\mathrm{He}_{1} V_{1}$ is given by the He atom slightly off $O$ site, as it presented in Fig. 2 with the formation energy of $4.47 \mathrm{eV}$. The $S$ configuration is higher in energy with $0.21 \mathrm{eV}$. In Ta, the $S$ site is the only found stable configuration. In this Ta case, all calculations having as the initial position as $T$ and $O$ position of He are finally relaxed into the $S$ configuration.

In the most stable configuration of clusters with 2 He atoms, $\mathrm{He}_{2} V_{1}$, Helium atoms and the vacancy are aligned along the $\langle 111\rangle$ direction for both metals, $\mathrm{V}$ and $\mathrm{Ta}$. In case of $\mathrm{He}_{3} V_{1}$ cluster, the He atoms form an equilateral triangle centered on the vacancy in $\mathrm{V}$, while in Ta, one He atom is shifted toward the vacancy and the 2 others occupy positions close to $O$ sites (Fig. 2c). The geome- try of clusters with the lowest formation energy from $n=4$ to $n=6$ have all He atoms close to $O$ sites in both $\mathrm{V}$ and Ta case.

In $\mathrm{V}$, for $\mathrm{He}_{1} \mathrm{~V}_{2}$ (Fig. 3), the $1 \mathrm{nn}$ configuration has higher formation energy than in the $2 n n$ configuration, as in the case of $V_{2}$. However, the difference is reduced to $0.09 \mathrm{eV}$ compared to 0.19 $\mathrm{eV}$ in the pure di-vacancy cluster (Table 1 ). Adding more He around the di-vacancy, the relative stability of mixed configurations $\mathrm{He}_{n} V_{2}$, having the di-vacancy in $1 n n$ and $2 n n$ positions, is reversed. For $n \geqslant 2$ the formation energy of the $1 n n$ configuration becomes lower than the corresponding formation energy in the $2 n n$ position. In Ta, except for the case of di-vacancy without He, the formation energy of $\mathrm{He}_{n} V_{2}^{1 n n}$ clusters is systematically lower than for the $\mathrm{He}_{n} V_{2}^{2 n n}$. Furthermore, the energy difference between these two cluster types is larger when adding more He atoms.

In general, comparing the energy landscape of $\mathrm{He}$ in the neighborhood of the vacancy in $\mathrm{V}$ and Ta, it can be noted that the $S$ site is attractive in Ta while it is repulsive in V (Figs. 2 and 3). A similar result has been found in a previous work [20]. This tendency is explained by a larger charge density in vacancy sites in $\mathrm{V}$ than in Ta. Vacancy charge density has a repulsive interaction with the atomic charge density of He. So He will have a stronger repulsive interaction at $S$ site in $\mathrm{V}$, increasing the formation energy enough to make this configuration unstable. As a result in the most stable configuration, He is shifted toward $O$ site.

In order to investigate the energetical cost to form $\mathrm{He}_{n+1} V_{m}$ clusters, we take for each cluster type the lowest formation energy found in our sampling. For all types of clusters $\mathrm{He}_{n} V_{m}$, the cost in energy to form $\mathrm{He}_{n+1} V_{m}$ increases linearly with $n$ but the slope decreases with $m$ (see Fig. 4). Due to random sampling of $\mathrm{He}_{n} V_{m}$ configuration, for given value of $m=2,3$, there are some uncertainties in the slope. The acceptable range in the value of the slope are marked in Fig. 4. The linear increase of formation energy with $n$ can be explained by the fact that a He atom is weakly bound to $\mathrm{He}_{n}$ clusters whereas He-vacancy binding energy is 1-2 orders of magnitude larger (Table 2). Hence, adding a He atom in the vacancy neighborhood results in adding one more He-vacancy interaction, until the vacancy is filled with He. The slope decreases with $m$ since $\mathrm{He}$ atoms have more space to maximize He-He distance. According to [19], for group $V B$ metals such as V and Ta, He-He interaction is weaker than for group VIB of transition metal (W, Mo) while the He-He distance is larger. As a consequence, for smaller clusters, one can expect a clearer inflection in the linear law for metals of group VIB than for group VB. 


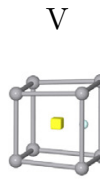

$\mathrm{Ta}$

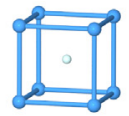

(a)

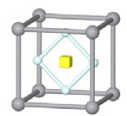

(d)

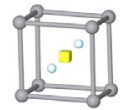

(b)

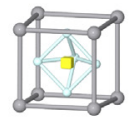

(e)
$\mathrm{V}$

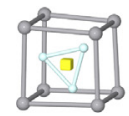

(c)
$\mathrm{Ta}$

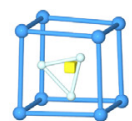

c)

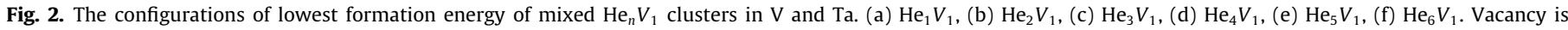

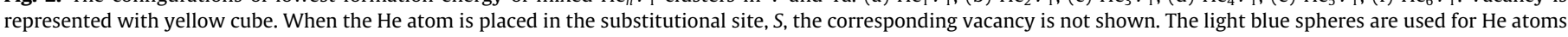

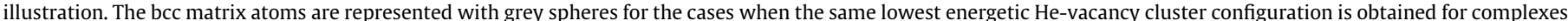

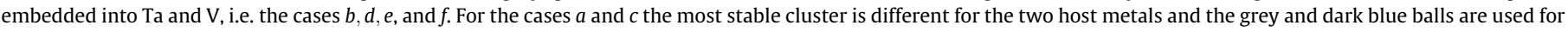
$\mathrm{V}$ or Ta atoms. (For interpretation of the references to colour in this figure legend, the reader is referred to the web version of this article.)
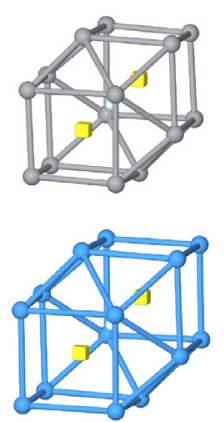

(a)
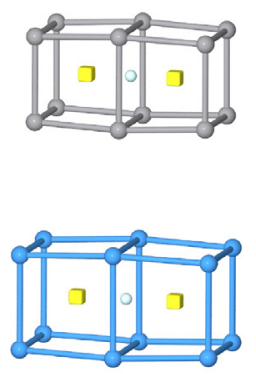

(e)
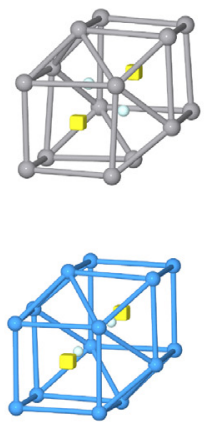

(b)
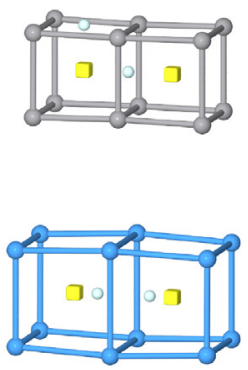

(f)
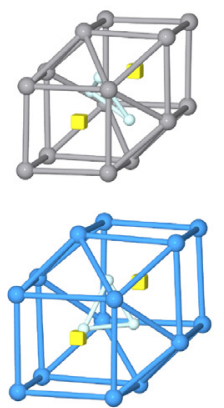

(c)
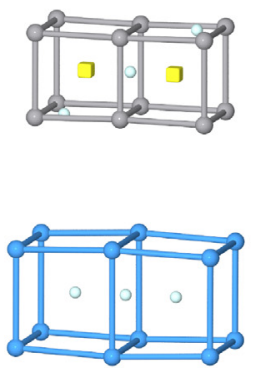

$(\mathrm{g})$
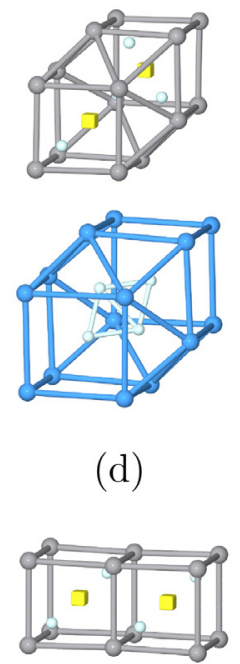

(d)

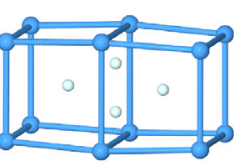

(h)

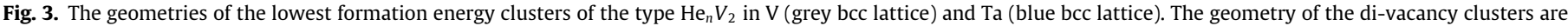

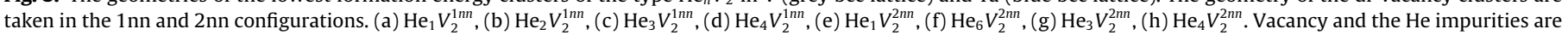

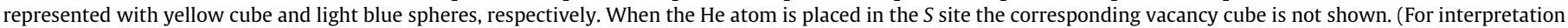
of the references to colour in this figure legend, the reader is referred to the web version of this article.)

\subsection{Stability and binding energies of He-vacancy clusters}

We investigated the stability of pure He and vacancy clusters and He-vacancy complexes looking at their binding energy to helium atom and mono-vacancy given respectively by:

$E_{\mathrm{He}-\mathrm{He}_{n} V_{m}}^{b}=E_{\mathrm{He}_{I}}+E_{\mathrm{He}_{n-1} V_{m}}-E_{\mathrm{He}_{n} V_{m}}-E_{0}$

and

$E_{V-\mathrm{He}_{n} V_{m}}^{b}=E_{V_{1}}+E_{\mathrm{He}_{n} V_{m-1}}-E_{\mathrm{He}_{n} V_{m}}-E_{0}$, where $E_{\mathrm{He}_{1}}$ is the total energy of the bcc supercell in which is inserted an interstitial He at $T$ or $S$ site for $\mathrm{V}$ and $\mathrm{Ta}$, respectively. $E_{V_{1}}$ is the total energy of the bcc supercell with one monovacancy. Binding energy of He/vacancy is the amount of energy necessary to escape from cluster. Following Eqs. (4) and (5), positive binding energy corresponds to an attractive interaction for He and vacancy, respectively. Therefore, clusters with the highest binding energy are more stable.

Fig. 5 emphasizes a general overview of the binding energy of one He atom in clusters $\mathrm{He}_{n} V_{m}$ for V and Ta. All clusters that have been considered (not only the most stable ones) are shown. Firstly, 
V

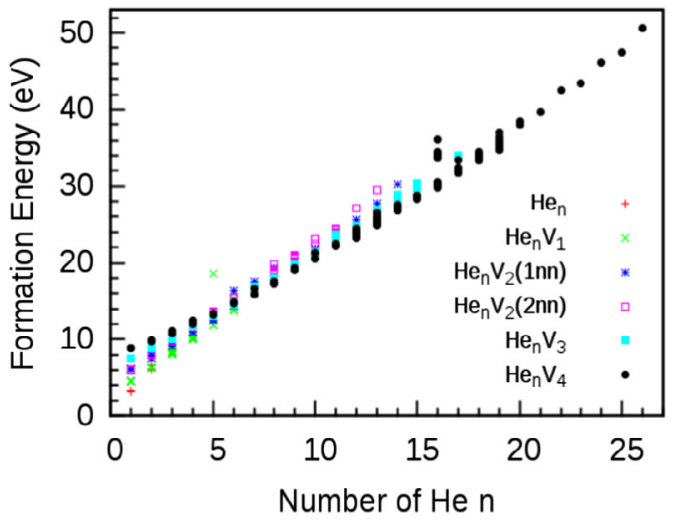

$\mathrm{Ta}$

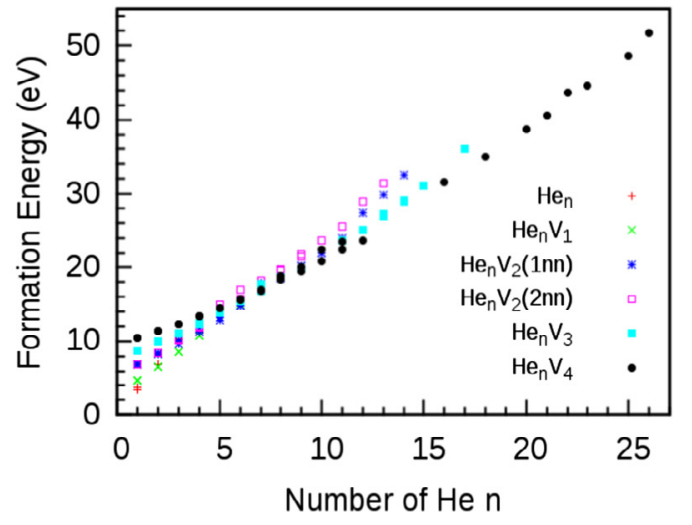

\begin{tabular}{|c|c|c|c|c|c|c|c|}
\hline & $\mathrm{m}$ & 0 & 1 & $2(1 \mathrm{nn})$ & $2(2 \mathrm{nn})$ & 3 & 4 \\
\hline $\mathrm{V}$ & $\Delta \mathrm{E}_{n \rightarrow n+1}^{f}(\mathrm{eV})$ & 2.97 & $1.86-2.05$ & $1.76-1.82$ & $1.81-1.88$ & 1.63 & $1.51-1.66$ \\
\hline $\mathrm{Ta}$ & $\Delta \mathrm{E}_{n \rightarrow n+1}^{f}(\mathrm{eV})$ & 3.26 & 2.06 & $1.73-2.19$ & $1.87-2.10$ & $.41-1.90$ & $1.07-1.35$ \\
\hline
\end{tabular}

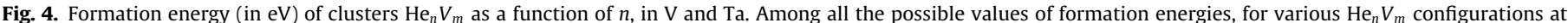

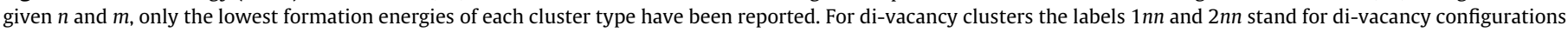

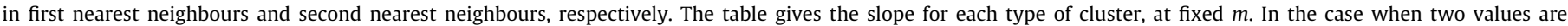
presented, we give the upper and lower limit of possibles choices of slope.

Table 2

The upper limit of binding energy (in eV) of He to $\mathrm{He}_{n} V_{m}$ clusters, extracted from the present calculations, using Eq. 4 , for small values of $n$ and $m$.

\begin{tabular}{|c|c|c|c|c|c|c|c|c|c|c|c|c|}
\hline \multirow{2}{*}{$\mathrm{n}$} & \multicolumn{6}{|c|}{$\mathrm{V}$} & \multicolumn{6}{|c|}{$\mathrm{Ta}$} \\
\hline & 0 & 1 & $2(1 \mathrm{nn})$ & $2(2 n n)$ & 3 & 4 & 0 & 1 & $2(1 \mathrm{nn})$ & $2(2 \mathrm{nn})$ & 3 & 4 \\
\hline 1 & 0.01 & 1.12 & 1.64 & 1.50 & 2.01 & 2.32 & 0.01 & 1.75 & 2.22 & 1.95 & 2.66 & 2.97 \\
\hline 2 & 0.09 & 1.34 & 1.73 & 1.24 & 1.81 & 2.21 & 0.06 & 1.62 & 2.00 & 1.98 & 2.25 & 2.58 \\
\hline 3 & 0.14 & 1.24 & 1.49 & 1.33 & 1.94 & 2.10 & 0.30 & 1.32 & 2.11 & 1.77 & 2.33 & 2.49 \\
\hline 4 & 0.24 & 1.26 & 1.39 & 1.38 & 1.48 & 2.04 & 0.20 & 1.29 & 1.92 & 1.72 & 2.25 & 2.42 \\
\hline 5 & & 1.23 & 1.58 & 1.16 & 1.87 & 1.85 & & & 1.84 & 1.07 & 1.93 & 2.31 \\
\hline 6 & & 1.30 & 1.16 & 1.51 & 1.50 & 1.57 & & & 1.49 & 1.95 & 1.87 & 2.29 \\
\hline
\end{tabular}
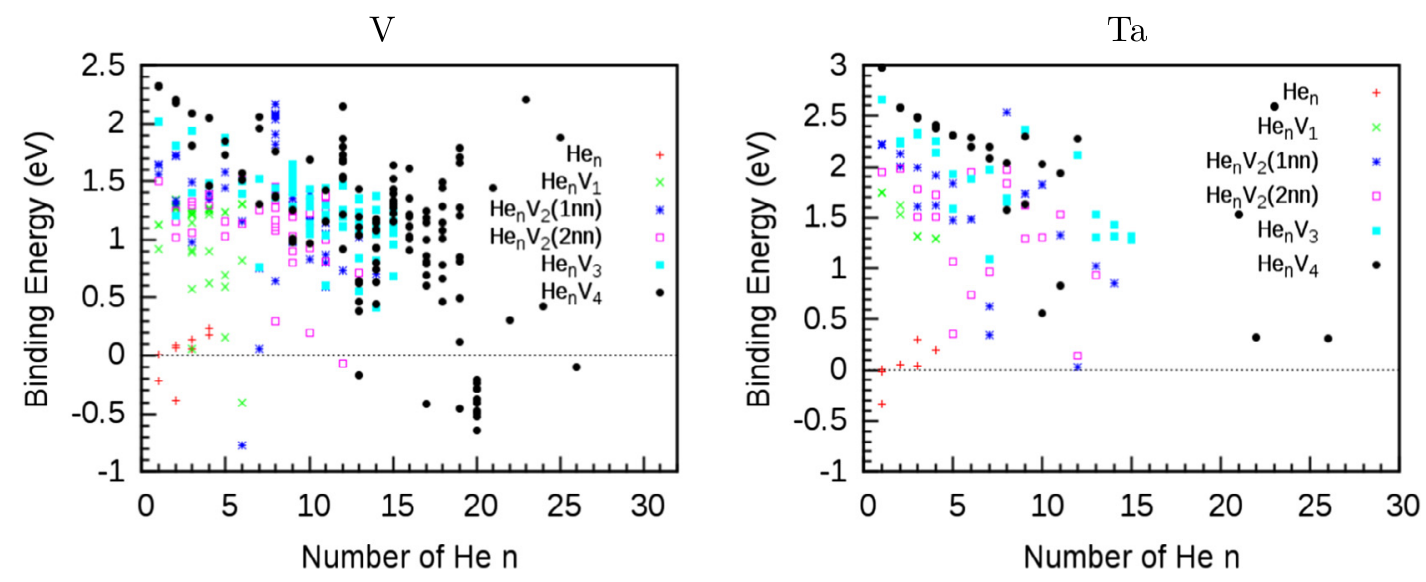

Fig. 5. Binding energy of He to $\mathrm{He}_{n} V_{m}$ clusters as a function of $n$ in $\mathrm{V}$ (left panel) and Ta (right panel).

it should be noted that the distribution in He binding energy for the same type of cluster, i.e. fixed number of $n$ and $m$, is rather broad. The scattered values of the binding energy indicate a strong dependence on the cluster morphology. For each pair of $n$ and $m$ there is a wide variety of clusters in which He is strongly or weakly bound. For practical use, e.g. kinetic simulations such as Object/
Event Kinetic Monte Carlo [44] or cluster dynamics simulations $[45,46]$, differences of cluster free energies, taking into account configurational entropy, should be used to get proper agreement with Atomistic Kinetic Monte Carlo [45,47]. As a first approximation, configurational entropy can be ignored and this binding free energy can be written as a difference of energies of most stable 
configurations. In $\mathrm{V}$, considering the most stable clusters, the binding energy of $\mathrm{He}$ in pure $\mathrm{He}_{n}$ clusters ranges from $0.01 \mathrm{eV}$ to 0.24 $\mathrm{eV}$, slowly increasing with the amount of He at least up to $n=4$. A similar behaviour is obtained in the case of Ta. We note that in $\mathrm{V}$ and $\mathrm{Ta}$ the binding energy of $\mathrm{He}$ in $\mathrm{He}_{4}$ clusters is almost 4 times lower than for the similar clusters in $\mathrm{Fe}(\sim 1 \mathrm{eV})$ [33]. Adding more $\mathrm{He}$ in interstitial clusters $\mathrm{He}_{n}$ at least up to $n=4$, amplifies lattice distortion so that, the increasing pressure may lead various processes such as the creation of Frenkel pair as predicted in Fe [4850] and observed in $\mathrm{Ni}$ [51] and $\mathrm{Au}$ [52]. However, is this conclusions should be supported only by supplementary calculations, such as interstitial energetic landscape, which are beyond the goals of this paper.

The upper limit of the He binding energy in $\mathrm{He}_{n} V_{m}$, at given $n$, increases with the number of vacancies. This tendency can be remarked from Table 2. A second trend is that for given $m$, the He binding energy seems to decrease with $n$, especially for $V_{3}$ (Fig. 5) With the increase of $n$ we expect an increase in the He pressure inside the cavity. Therefore, spontaneous emission of He or self-interstitial, $\mathrm{V}$ or Ta atoms, may occur for large $\mathrm{n} / \mathrm{m}$ ratio $[53,54]$. This behaviour has been observed at low temperature by Fedorov [55] in V and V alloys, and also theoretically predicted in Fe by Morishita [49].

However, a closer look at Fig. 5 reveals that for $m=1$, in V, the binding energy seems to saturate (up to $n=6$ ) indicating that more He atoms can be added before internal pressure reaches a threshold such that significant drop in the binding energy occurs. The present observations are in agreement with previous calculations which have showed that the mono-vacancy clusters containing tens of $\mathrm{He}$ atoms still have attractive interaction with $\mathrm{He}$ in $\mathrm{V}$ [56]. This apparent drop in the binding energy with $n$ may reflect the fact we missed more stable clusters in our sampling. In the di-vacancy case, we see that He makes the $\mathrm{He}_{n} V_{2}^{1 n n}$ clusters more stable than the $\mathrm{He}_{n} V_{2}^{2 n n}$ ones whereas the opposite situation is observed without $\mathrm{He}$. In both $\mathrm{V}$ and $\mathrm{Ta}, \mathrm{He}_{8} V_{2}^{1 n n}$ seems particularly stable compared to $\mathrm{He}_{7} V_{2}^{1 n n}$ and $\mathrm{He}_{9} V_{2}^{1 n n}$. In the $\mathrm{He}_{8} V_{2}^{1 n n}$ cluster He atoms occupy high symmetry interstitial sites $O$ and $T$ while the position of He is more shifted from $O$ and $T$ sites in $\mathrm{He}_{7} V_{2}^{1 n n}$ and $\mathrm{He}_{9} V_{2}^{1 n n}$. Likewise, in $\mathrm{V}, \mathrm{H}_{20} V_{4}$ clusters are unstable, contrary to $\mathrm{He}_{19} V_{4}$ and $\mathrm{He}_{21} V_{4}$ clusters for which more high symmetry sites are occupied by He atoms.

The binding energy of a vacancy to pure vacancy clusters $V_{m}$ increases with the number of vacancies from $0.27 \mathrm{eV}$ for $m=2$ to $0.88 \mathrm{eV}$ for $m=4 \mathrm{in} \mathrm{V}$ (Fig. 6). It seems to reach a plateau at $m=4$ around $0.87 \mathrm{eV}$. Similar trend has been reported in Fe, using molecular dynamics simulations with different empirical poten- tials, for which the binding energy saturates slightly above $1 \mathrm{eV}$ for $m \gtrsim 20$ [49].

From the values presented in Table 3, the presence of He atoms in vacancy clusters enhances the binding energy to mono-vacancy, reflecting the fact that He stabilizes vacancy type clusters $[33,49]$. We find again $\mathrm{He}_{n} V_{2}^{1 n n}$ are more stable than $\mathrm{He}_{n} V_{2}^{2 n n}$ for $\mathrm{n} \geqslant 2$ and $\mathrm{n}$ $\geqslant 1$ in $\mathrm{V}$ and in $\mathrm{Ta}$, respectively.

\subsection{Migration energy barriers}

The diffusion processes of interstitial He and vacancy are important in order to understand the mechanism of cluster nucleation and growth during the primary stages. In the following, we restrict the scope of migration of vacancies toward their 1st nearest neighbour, i.e. in $\langle 111\rangle$ direction. To reveal the minimum energy path (MEP) of migrations mechanism involving vacancyHe complexes is not obvious. We have tried two steps procedure. Firstly, we divided the unrelaxed path between the initial and final configuration in several segments. The most stable configurations found along these segments were taken as intermediate steps in order to precondition the finding MEP algorithm. Secondly, by fixing the initial and final configurations we let the minimization algorithm to find the MEP. We retain the path with the lowest energy barrier.

\subsubsection{Vanadium}

The interstitial He diffusion is studied by examination of two paths which connect the lowest energy configurations: $T$ site to $T$ site, and $T$ site to $O$ site. The $T \rightarrow T$ mechanism corresponds to a migration barrier of $0.12 \mathrm{eV}$ in $\mathrm{V}$ (Table 4). This migration barrier is in good agreement with TDS experiments for which the value of $0.13 \mathrm{eV}$ is reported [55]. It should be noted that the migration barrier of this mechanism is twice as high as in Fe [33]. The migration barrier of the mechanism $T \rightarrow O$ is higher and has the value of $0.26 \mathrm{eV}$. Consequently, the interstitial He prefers to diffuse via first neighbor $T$ sites. The same conclusion was drawn in Fe [33].

The DFT migration barrier of the mono-vacancy is found equal to $0.48 \mathrm{eV}$. This value is in agreement with TDS experiments of Fedorov [55], $0.5 \mathrm{eV}$, and van Veen, $0.47 \mathrm{eV}$ [57]. The di-vacancy migration barrier from the $V_{2}^{1 n n}$ state (Fig. 1b) to $V_{2}^{2 n n}$ configuration (Fig. 1c) is asymmetrical, with $0.34 \mathrm{eV}$ and $0.58 \mathrm{eV}$ for forward and reverse jumps, respectively. Another possible migration of divacancy is the direct mechanism path from a $V_{2}^{2 n n}$ to another $V_{2}^{2 n n}$ configuration. This barrier has the value of $0.37 \mathrm{eV}$. This divacancy mechanism implies two migration steps by dissociationassociation. One of the vacancy dissociates from the $V_{2}^{2 n n}$ cluster
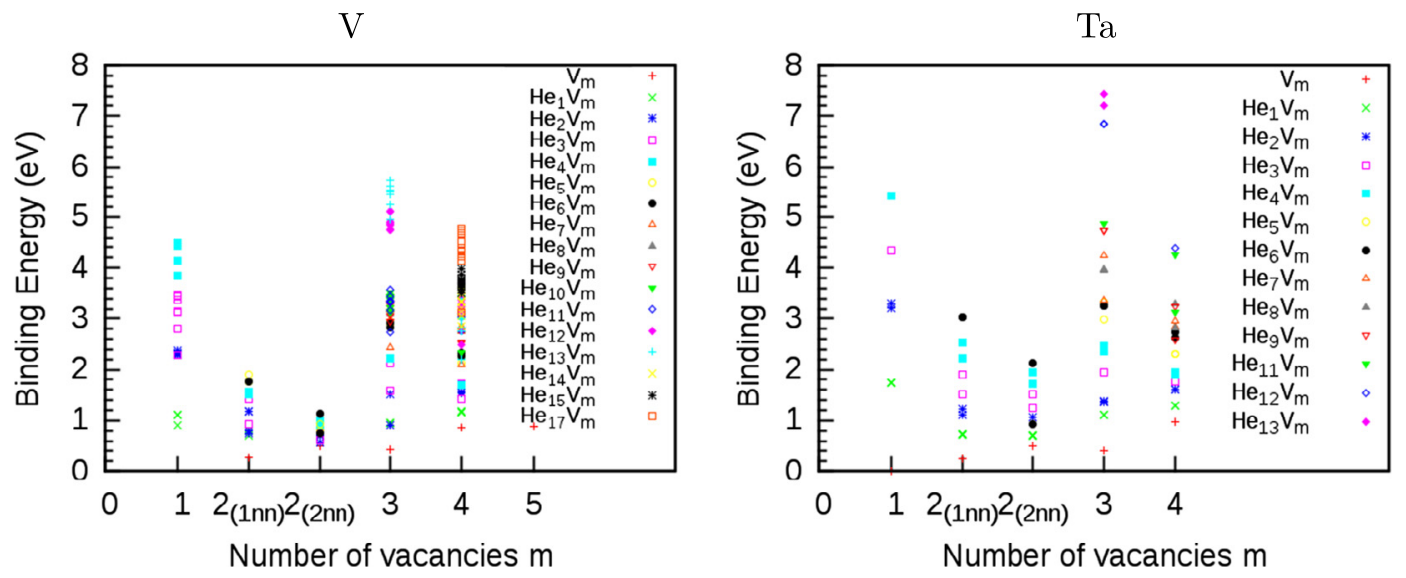

Fig. 6. Binding energy of vacancy, $V_{1}$, to $\mathrm{He}_{n} V_{m}$ clusters as a function of $m$ in $\mathrm{V}$ (left panel) and Ta (right panel). 
Table 3

The upper limit of binding energy (in eV) of vacancy to $\mathrm{He}_{n} V_{m}$ clusters, extracted from the present calculations, using Eq. (5), small values of $n$ and $m$.

\begin{tabular}{|c|c|c|c|c|c|c|c|c|c|c|c|}
\hline \multirow{3}{*}{$\mathrm{n}$} & \multicolumn{6}{|c|}{$\mathrm{V}$} & \multicolumn{5}{|c|}{$\mathrm{Ta}$} \\
\hline & \multicolumn{11}{|c|}{$\mathrm{m}$} \\
\hline & 1 & $2(1 \mathrm{nn})$ & $2(2 n n)$ & 3 & 4 & 5 & 1 & $2(1 \mathrm{nn})$ & $2(2 n n)$ & 3 & 4 \\
\hline 0 & 0.00 & 0.27 & 0.51 & 0.43 & 0.87 & 0.88 & 0.00 & 0.26 & 0.50 & 0.40 & 0.97 \\
\hline 1 & 1.11 & 0.79 & 0.89 & 0.94 & 1.17 & & 1.74 & 0.73 & 0.70 & 1.11 & 1.28 \\
\hline 2 & 2.37 & 1.19 & 0.79 & 1.50 & 1.57 & & 3.31 & 1.11 & 1.06 & 1.39 & 1.61 \\
\hline 3 & 3.47 & 1.43 & 0.88 & 2.12 & 1.72 & & 4.33 & 1.90 & 1.51 & 1.94 & 1.77 \\
\hline 4 & 4.49 & 1.56 & 1.00 & 2.22 & 2.28 & & 5.43 & 2.52 & 1.94 & 2.47 & 1.93 \\
\hline 5 & & 1.91 & 0.92 & 2.93 & 2.25 & & & & & 3.33 & 2.32 \\
\hline 6 & & 1.76 & 1.13 & 2.92 & 2.32 & & & 3.02 & 2.14 & 3.26 & 2.73 \\
\hline
\end{tabular}

Table 4

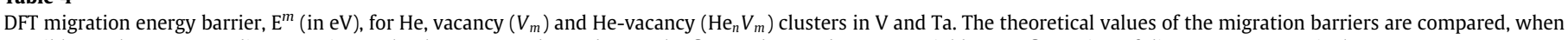
possible, to the corresponding experimental values. $1 \mathrm{nn}$ and $2 \mathrm{nn}$ denote the first and second nearest neighbor configuration of di-vacancy, respectively.

\begin{tabular}{|c|c|c|c|c|c|c|}
\hline \multirow[t]{2}{*}{ Configuration } & \multicolumn{3}{|c|}{$\mathrm{V}$} & \multicolumn{3}{|c|}{ Ta } \\
\hline & Path & $\mathrm{E}^{m}(\mathrm{eV})$ & Exp. (eV) & Path & $\mathrm{E}^{m}(\mathrm{eV})$ & Exp. (eV) \\
\hline \multicolumn{7}{|l|}{ He interstitial } \\
\hline \multirow[t]{2}{*}{$\mathrm{He}$} & $T \rightarrow T$ & 0.12 & $0.13[55]$ & $T \rightarrow T$ & 0.18 & \\
\hline & $T \rightarrow O$ & 0.26 & & $T \rightarrow O$ & 0.40 & \\
\hline \multicolumn{7}{|l|}{$V_{m}$ clusters } \\
\hline Mono-vacancy & $1 \mathrm{nn}$ & 0.48 & $\begin{array}{c}0.5[55,60] \\
0.47[57]\end{array}$ & $1 \mathrm{nn}$ & 0.72 & $0.7[61,60]$ \\
\hline Di-vacancy & $1 \mathrm{nn} \rightarrow 2 \mathrm{nn}$ & 0.34 & & $1 \mathrm{nn} \rightarrow 2 \mathrm{nn}$ & 0.63 & \\
\hline Di-vacancy & $2 \mathrm{nn} \rightarrow 1 \mathrm{nn}$ & 0.58 & & $2 \mathrm{nn} \rightarrow 1 \mathrm{nn}$ & 0.87 & \\
\hline Di-vacancy & $2 \mathrm{nn} \rightarrow 2 \mathrm{nn}$ & 0.37 & & $2 \mathrm{nn} \rightarrow 2 \mathrm{nn}$ & 0.55 & \\
\hline \multicolumn{7}{|l|}{$\mathrm{He}_{n} V_{m}$ clusters } \\
\hline $\mathrm{He}_{1} V_{1}$ & $1 \mathrm{nn}$ & 1.27 & & $1 \mathrm{nn}$ & 1.89 & \\
\hline $\mathrm{He}_{1} V_{2}$ & $1 \mathrm{nn} \rightarrow 2 \mathrm{nn}$ & 0.79 & & $1 \mathrm{nn} \rightarrow 2 \mathrm{nn}$ & 1.14 & \\
\hline $\mathrm{He}_{1} V_{2}$ & $2 \mathrm{nn} \rightarrow 1 \mathrm{nn}$ & 0.88 & & $2 \mathrm{nn} \rightarrow 1 \mathrm{nn}$ & 1.12 & \\
\hline $\mathrm{He}_{1} V_{2}$ & $2 \mathrm{nn} \rightarrow 2 \mathrm{nn}$ & 0.66 & & $2 \mathrm{nn} \rightarrow 2 \mathrm{nn}$ & 1.12 & \\
\hline
\end{tabular}

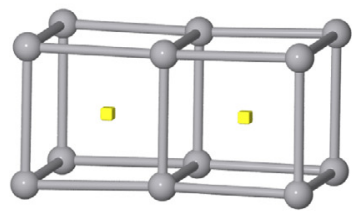

(a)

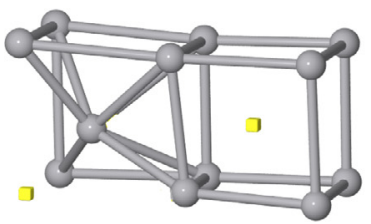

(b)

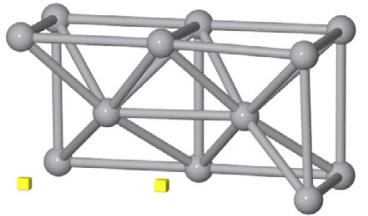

(c)

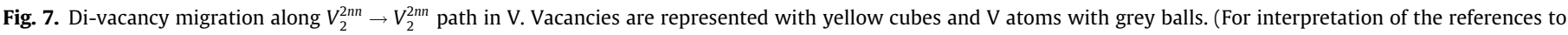
colour in this figure legend, the reader is referred to the web version of this article.)

to reach its 1 st nearest neighbour position along the $\langle 111\rangle$ direction. At this point the two vacancies are in a 4 th nearest neighbour configuration (Fig. 7b). Then the second vacancy that jump along $\langle 111\rangle$ direction and associates with the first vacancy to form again the $V_{2}^{2 n n}$ cluster (Fig. 7c). It should be mentioned that a direct jump from configuration presented in Fig. 7a to configuration of Fig. 7c dissociates similarly, following the mechanism described above.

The influence of He on vacancy diffusion is considered using the same NEB algorithm. As in previous cases the minimum energy path of $\mathrm{HeV}_{1}$ cluster is searched by fixing the initial and final states in the corresponding lowest energy configurations. We found that the migration of $\mathrm{HeV}_{1}$ cluster is a two steps mechanism: (i) the $\mathrm{He}$ atom jumps from initial $O$ site to final 3rd nearest neighbour $O$ site, hopping through 1 st nearest $T$ sites (this mechanism is schematized in Fig. 8a-c) and (ii) the vacancy migrates along $\langle 111\rangle$ direction and He hops from $T$ to $O$ site to reach their final position (Fig. 8d and e). The computed DFT migration barrier is about $1.27 \mathrm{eV}$, which corresponds roughly to the He-vacancy binding energy plus migration barrier of $\mathrm{He}(T \rightarrow T)$. As a result, $\mathrm{He}$ increases the diffusion barrier of mono-vacancy by $0.67 \mathrm{eV}$. This dissociative mechanism has been also experimentally inferred $[58,59]$ from He implanted depth profiles and He thermal desorption experiments at low temperature.

The di-vacancy in the presence of He can migrate from $\mathrm{He}_{1} V_{2}^{1 \text { nn }}$ into $\mathrm{He}_{1} V_{2}^{2 n n}$ configuration by passing a $0.79 \mathrm{eV}$ barrier. Migration from $\mathrm{He}_{1} V_{2}^{2 n n}$ to $\mathrm{He}_{1} V_{2}^{2 n n}$ configuration has a lower barrier (0.66 $\mathrm{eV}$ ) compared to the previous $\mathrm{He}_{1} V_{2}^{1 n n} \rightarrow \mathrm{He}_{1} V_{2}^{2 n n}$ path. The $\mathrm{He}_{1} V_{2}^{2 n n} \rightarrow \mathrm{He}_{1} V_{2}^{2 n n}$ process is a two steps mechanism which is schematized in Fig. 9a-d. Firstly, one vacancy of the cluster dissociates to reach first nearest neighbour site in the $\langle 111\rangle$ direction. The geometry of the cluster is changed into a slightly shifted $V_{2}^{1 n n}$ configuration (Fig. 9b). Secondly, the second vacancy moves to its nearest neighbour in the same $\langle 111\rangle$ direction. Simultaneously the He atom jumps to final $O$ position between the two vacancies, similar to the initial configuration (Fig. 9c-e). The trapped He atom roughly doubles the migration barrier of the di-vacancy for both $V_{2}^{1 n n} \rightarrow V_{2}^{2 n n}$ and $V_{2}^{2 n n} \rightarrow V_{2}^{2 n n}$ paths, and increases the barrier by 


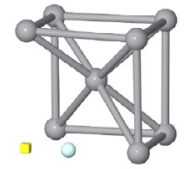

(a)

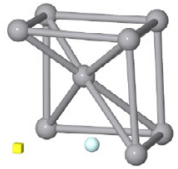

(b)

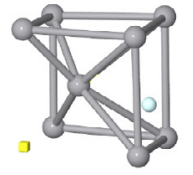

(c)

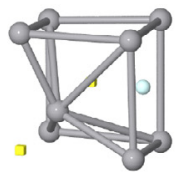

(d)

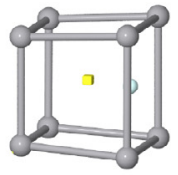

(e)

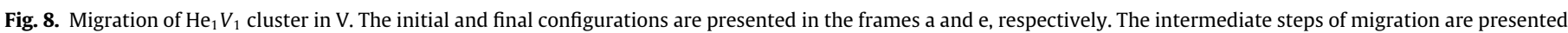

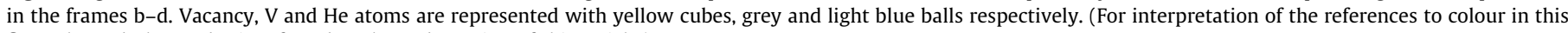
figure legend, the reader is referred to the web version of this article.)

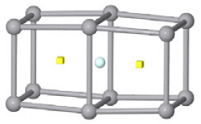

(a)

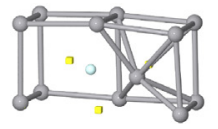

(b)

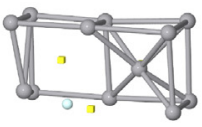

(c)

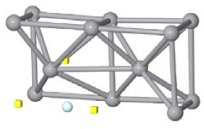

(d)

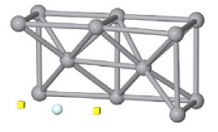

(e)

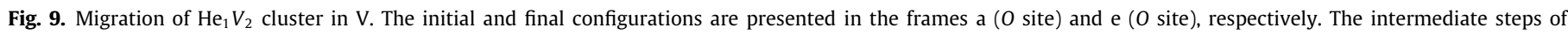

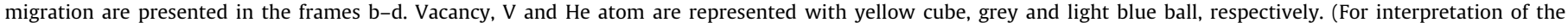
references to colour in this figure legend, the reader is referred to the web version of this article.)

$50 \%$ of the $V_{2}^{2 n n} \rightarrow V_{2}^{1 n n}$ path. In the presence or not of the He the barrier energy of mono-vacancy remains higher than for divacancy. However, the presence of He atom increases the gap between mono-vacancy and di-vacancy energy barrier.

\subsubsection{Tantalum}

The migration barrier of interstitial He in Ta lattice is reported in Table 4. The migration barrier of interstitial He are $0.18 \mathrm{eV}$ and $0.40 \mathrm{eV}$ for the $T \rightarrow T$ and $T \rightarrow O$ paths, respectively. Therefore the lowest energy migration mechanism corresponds to the $T \rightarrow T$, similar to the $\mathrm{V}$ case.

The value of migration barrier of mono-vacancy in Ta is $0.72 \mathrm{eV}$. This result is consistent with resistivity recovery experiments which give the value of $0.7 \mathrm{eV}$ [61]. The lowest migration barrier of di-vacancy, with a value of $0.55 \mathrm{eV}$, corresponds to the $V_{2}^{2 n n} \rightarrow$ $V_{2}^{2 n n}$ path. This is similar to $\mathrm{V}$ case for which this mechanism is more favorable than the migration of the mono-vacancy. These results show a different behaviour compared to $\mathrm{Fe}[21]$ and $\mathrm{W}$ [42] for which the energy barrier of mono-vacancy and divacancy are very close.

Diffusion of mixed cluster $\mathrm{He}_{1} V_{1}$ involves firstly the dissociation of He from the vacancy to reach the 1 st nearest $T$ site (Fig. 10b). Subsequently, the He atom hops via $T$ interstitial sites. Simultaneously, the vacancy jumps to first nearest neighbour site (Fig. 10c and d). Finally, He recombines with the mono-vacancy at $S$ site (Fig. 10e). The mechanism described above corresponds to the impeded interstitial mechanism or He-vacancy dissociative mechanism $[60,62]$ where a He atom diffuses through interstitial sites after dissociation from one vacancy and before being retrapped by another vacancy. It leads to an increase of the migration barrier of mono-vacancy by $1.17 \mathrm{eV}$. Diffusion of $\mathrm{He}_{1} V_{2}$ clusters occurs according to the same scenario described above. As for the monovacancy, an increase of the migration barrier is obtained. However, for this case, the increase of migration energy due to $\mathrm{He}$ is twice smaller $(0.51 \mathrm{eV})$ for $\mathrm{He}_{1} V_{2}^{1 n n} \rightarrow \mathrm{He}_{1} V_{2}^{2 n n}$ path than for $\mathrm{He}_{1} V_{1}$ case. Contrary to the $\mathrm{V}$ case, all paths involving $\mathrm{He}_{1} V_{2}$ clusters have almost the same barrier in presence of He. Consequently, $\mathrm{He}_{1} V_{2}$ clusters can migrate via these three channels.

Thermal desorption spectra experiments give direct access to dissociation energy defined as the sum of binding energy and migration energy of He atom or vacancy [49]:

$E_{\mathrm{He} / V-\mathrm{He}_{n} V_{m}}^{d}=E_{\mathrm{He} / V-\mathrm{He}_{n} V_{m}}^{b}+E_{\mathrm{He} / V}^{m}$.

This is the amount of activation energy needed to expel He or vacancy from the cluster. In the previous equation, $E_{\mathrm{He} / V}$ denotes the corresponding energy related to He or vacancy. In Fig. 11 is shown, for $\mathrm{V}$ and $\mathrm{Ta}$, the dissociation energy of He and vacancy with respect to the lowest energy configuration of $\mathrm{He}_{n} V_{m}$ cluster as a function of $n / m$ ratio. This quantity allows to determine whether a mixed cluster tends to emit a He atom or a vacancy to gain stability, i.e when $\mathrm{E}_{\mathrm{He}}^{d}>\mathrm{E}_{V}^{d}$, the cluster is more likely to emit a vacancy. The presence of a crossover between dissociation energies of He or vacancy shows that the most stable clusters have a $n / m$ ratio of around 0.9 with $\mathrm{E}^{d} \sim 1.9 \mathrm{eV}$ in $\mathrm{V}$. This theoretical result is close to experimental results which indicate $\mathrm{E}^{d}=1.66 \mathrm{eV}$ [59]. The fact that the most stable clusters are such that $n \approx m$ is consistent with

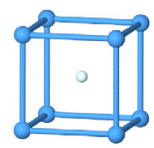

(a)

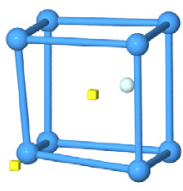

(b)

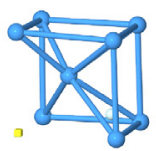

(c)

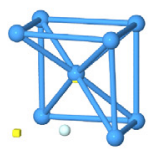

(d)

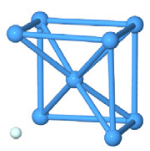

(e)

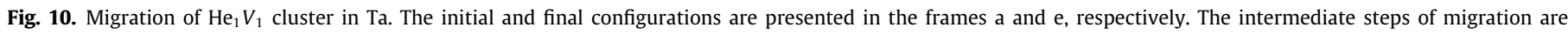

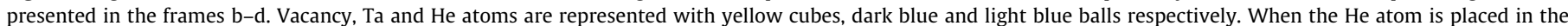

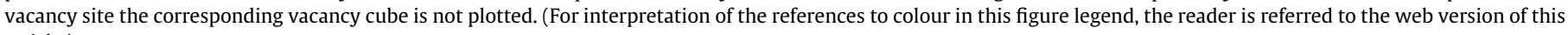
article.) 
V

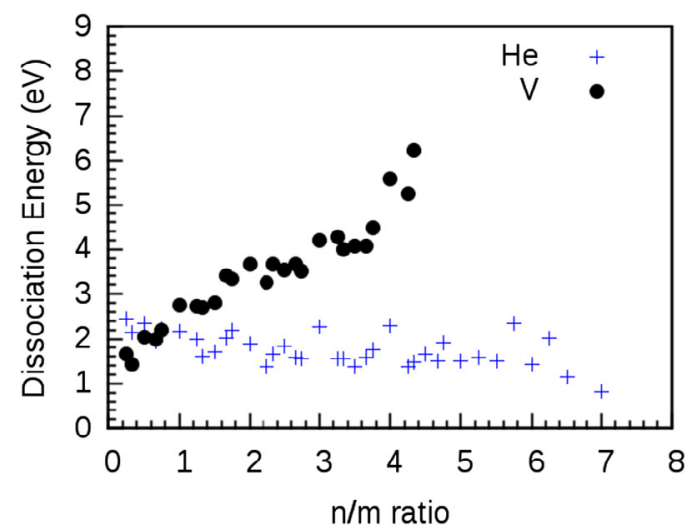

Ta

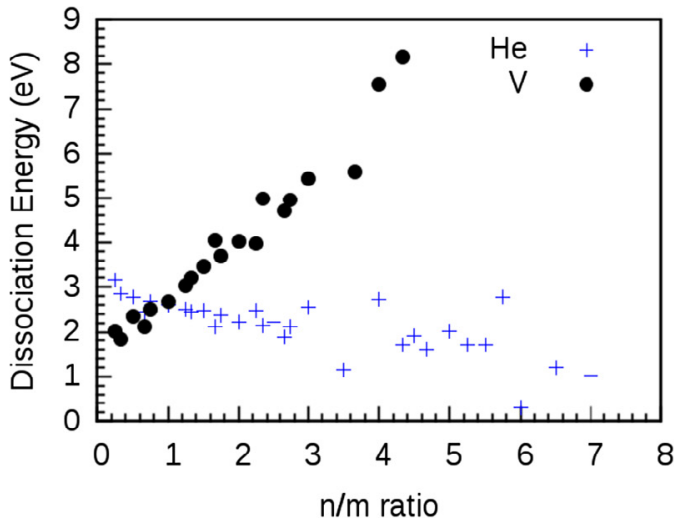

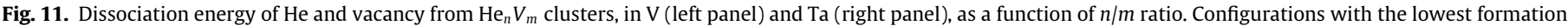
energy are taken as references.

hypotheses taken to analyse TDS experiments [55] and previous results in Fe [33]. The corresponding quantities for Ta are estimated to $\sim 0.9 \mathrm{n} / \mathrm{m}$ ratio and $\mathrm{E}^{d} \sim 2.5 \mathrm{eV}$.

\section{Conclusion}

We performed first-principle calculations of the formation, binding and migration energy of vacancies in the presence or not of He atoms in bcc V and Ta. In summary, we present our main results:

- interstitial He atoms diffuse through tetrahedral sites in both $\mathrm{V}$ and $\mathrm{Ta}$, as in the case of $\mathrm{W}[38,63]$.

- for both $\mathrm{V}$ and Ta, the formation energy of di-vacancy in $V_{2}^{2 n n}$ configuration is lower than the formation energy of $V_{2}^{1 n n}$ configuration. This tendency is also observed in the VI metals such as Mo and $\mathrm{W}$ and in Fe. The di-vacancies in $\mathrm{V}$ and Ta have a weak attractive binding energy whereas it is repulsive for group $V I$ metals.

- the effect of He on di-vacancies is to increase the formation energy of the $V_{2}^{1 n n}$ and $V_{2}^{2 n n}$ configurations. This increase is more important for $V_{2}^{2 n n}$ than $V_{2}^{1 n n}$ configuration so that the hierarchy is reversed, i.e. $E_{\mathrm{He}_{n} V_{2}^{2 n n}}^{f}>E_{\mathrm{He}_{n} V_{2}^{1 n n}}^{f}$ for $n \geqslant 2$ in $\mathrm{V}$ and $n \geqslant 1$ in Ta. In addition, the binding energy of $\mathrm{He}_{n} V_{2}^{1 n n}$ to $\mathrm{He}$ atom and mono-vacancy is larger than the binding energy of $\mathrm{He}_{n} V_{2}^{2 n n}$ to He atom and mono-vacancy when $n$ increases.

- as general tendency the He atom stabilizes vacancy type clusters and vice versa. This is a trend that was also observed in $\mathrm{W}$ [63].

- for the two present studied metals of group $V B$, di-vacancy is more mobile than mono-vacancy. The presence of He atom enhances the migration energy barrier of both mono-vacancy and di-vacancy but also their relative difference.

- in absence of He, di-vacancy diffuses preferentially via $V_{2}^{2 n n} \rightarrow$ $V_{2}^{2 n n}$ path in a 2 steps vacancy dissociation-association process in both $\mathrm{V}$ and $\mathrm{Ta}$.

- in the presence of He atom, diffusion of both mono-vacancy and di-vacancy involves dissociation of He atom from the vacancy type cluster following the 'impeded interstitial mechanism' in both $\mathrm{V}$ and Ta case. For the di-vacancy diffusion in $\mathrm{V}$, the $V_{2}^{2 n n}$ $\rightarrow V_{2}^{2 n n}$ path is still favored, but in case of Ta, all paths have the same energy barrier.
- the most stable clusters have a $n / m$ ratio about 0.9 with a dissociation energy of $1.9 \mathrm{eV}$ and $2.5 \mathrm{eV}$ for $\mathrm{V}$ and Ta, respectively. In the case of $\mathrm{W}$ the values are somehow different: the ratio $n / m$ is 2 and the dissociation energy is almost $4 \mathrm{eV}[38,64]$

The present study permits to get insight into the kinetics of the growth of $\mathrm{He}_{n} V_{m}$ clusters. The energy landscape of the He atoms in the bcc matrix of the $V$ and Ta metals are revealed with some particularities compared to others bcc metals. The results of this paper, are useful, can be used as parameters for kinetic modelling such as object/event kinetic Monte-Carlo or cluster dynamics simulations.

\section{Acknowledgements}

The authors acknowledge the support of the French Agence Nationale de la Recherche (ANR) under project EPigRAPH ANR14-CE07-0001. The authors acknowledge support from the GENCI - (CINES/CCRT/IDRIS) computer centre under Grant No. A0030906973. and from PRACE (Partnership for Advanced Computing in Europe) for access to the resource MareNostrumIII at the Barcelona supercomputing centre (TRANSOM project).

\section{References}

[1] G.R. Odette, M.J. Alinger, B.D. Wirth, Recent developments in irradiationresistant steels, Annu. Rev. Mater. Res. 38 (1) (2008) 471-503, https://doi.org/ 10.1146/annurev.matsci.38.060407.130315.

[2] S.J. Zinkle, L.L. Snead, Designing radiation resistance in materials for fusion energy, Annu. Rev. Mater. Res. 44 (1) (2014) 241-267, https://doi.org/10.1146/ annurev-matsci-070813-113627, ISSN 1531-7331, 1545-4118.

[3] S.J. Zinkle, Challenges in developing materials for fusion technology - past, present and future, Fusion Sci. Technol. 64 (2) (2013) 65, https://doi.org/ 10.13182/FST13-631.

[4] S.J. Zinkle, H. Matsui, D.L. Smith, A.F. Rowcliffe, E. van Osch, K. Abe, V.A Kazakov, Research and development on vanadium alloys for fusion applications, J. Nucl. Mater. 258 (1998) 205, https://doi.org/10.1016/S00223115(98)00269-4, ISSN 0022-3115, URL <http://www.sciencedirect.com/ science/article/pii/S0022311598002694>.

[5] T. Muroga, T. Nagasaka, A. Iiyoshi, A. Kawabata, S. Sakurai, M. Sakata, Nifs program for large ingot production of a V-Cr-Ti alloy, J. Nucl. Mater. 283 (2000) 711, https://doi.org/10.1016/S0022-3115(00)00281-6 (9th International Conference on Fusion Reactor Materials) ISSN 0022-3115. URL <http:// www.sciencedirect.com/science/article/pii/S0022311500002816>.

[6] R.J. Kurtz, K. Abe, V.M. Chernov, D.T. Hoelzer, H. Matsui, T. Muroga, G.R. Odette, Recent progress on development of vanadium alloys for fusion, J. Nucl. Mater. 329 (2004) 47, https://doi.org/10.1016/j.jnucmat.2004.04.299 (Proceedings of the 11th International Conference on Fusion Reactor Materials (ICFRM-11)) ISSN 0022-3115. URL <http://www.sciencedirect.com/science/article/pii/ S0022311504004295>.

[7] R.E. Gold, D.L. Harrod, Refractory metal alloys for fusion reactor applications, J. Nucl. Mater. 85 (1979) 805, https://doi.org/10.1016/0022-3115(79)90359-3. 
[8] M.A. Kirk, R.C. Birtcher, T.H. Blewitt, Measurements of neutron spectra and fluxes at spallation-neutron sources and their application to radiation effects research, J. Nucl. Mater. 96 (1981) 37, https://doi.org/10.1016/0022-3115(81) 90216-6.

[9] Y. Dai, G.R. Odette, T. Yamamoto, 1.06 - the effects of helium in irradiated structural alloys, in: Rudy J.M. Konings (Ed.), Comprehensive Nuclear Materials, Elsevier, Oxford, 2012, pp. 141-193, https://doi.org/10.1016/B9780-08-056033-5.00006-9, ISSN 978-0-08-056033-5. URL <http://www. sciencedirect.com/science/article/pii/B9780080560335000069>.

[10] H. Matsui, M. Tanaka, M. Yamamoto, M. Tada, Embrittlement of vanadium alloys doped with helium, J. Nucl. Mater. 191 (1992) 919-923, https://doi.org/ 10.1016/0022-3115(92)90607-M, ISSN 0022-3115. URL <http://www. sciencedirect.com/science/article/pii/002231159290607M>.

[11] D. Kaletta, Low-cycle irradiations of vanadium with 2 mev helium ions at elevated temperatures, J. Nucl. Mater. 76 (1978) 221-223, https://doi.org/ 10.1016/0022-3115(78)90143-5, ISSN 0022-3115. URL <http:// www.sciencedirect.com/science/article/pii/0022311578901435>.

[12] A.V. Federov, G.P. Buitenhuis, A. van Veen, A.I. Ryazanov, J.H. Evans, W. van Witzenburg, K.T. Westerduin, Helium desorption studies on vanadium and V5Ti and V-3Ti-1Si alloys and their relevance to helium embrittlement, J. Nucl. Mater. 227 (3) (1996) 312-321, https://doi.org/10.1016/0022-3115(95)001379, ISSN 0022-3115. URL <http://www.sciencedirect.com/science/article/pii/ 0022311595001379>.

[13] S.R. Bhattacharyya, T.K. Chini, D. Basu, Transmission electron microscope studies of tantalum irradiated by mev energy $\alpha$ particles, J. Mater. Sci. Lett. 16 (7)(1997) 577-579, https://doi.org/10.1023/A:1018578124469, ISSN 1573-4811.

[14] M. Heerschap, E. Schüller, B. Langevin, A. Trapani, Helium gas bubbles in vanadium, J. Nucl. Mater. 46 (2) (1973) 207-209, https://doi.org/10.1016/ 0022-3115(73)90136-0, ISSN 0022-3115. URL <http://www.sciencedirect. com/science/article/pii/0022311573901360>.

[15] J. Hua, Y-L. Liu, H-S. Li, M-W. Zhao, X-D. Liu, The role of alloying element on the behaviors of helium in vanadium: Ti as an example, Comput. Condens. Matter 3 (2015)1-8, https://doi.org/10.1016/j.cocom.2015.03.004, ISSN 2352-2143. URL <http://www.sciencedirect.com/science/article/pii/S235221431500012X>.

[16] P. Zhang, J. Zhao, Y. Qin, B. Wen, Stability and migration property of helium and self defects in vanadium and $\mathrm{V}-4 \mathrm{Cr}-4 \mathrm{Ti}$ alloy by first-principles, J. Nucl. Mater. 413 (2) (2011) 90-94, https://doi.org/10.1016/j.jnucmat.2011.03.031. ISSN 0022-3115. URL <http://www.sciencedirect.com/science/article/pii/ S0022311511002996>.

[17] P. Zhang, J. Zhao, Y. Qin, B. Wen, Stability and dissolution of helium-vacancy complexes in vanadium solid, J. Nucl. Mater. 419 (1-3)(2011) 1-8, https://doi. org/10.1016/j.jnucmat.2011.08.023, ISSN 0022-3115. URL <http:// www.sciencedirect.com/science/article/pii/S0022311511007999>.

[18] P. Zhang, T. Zou, J. Zhao, P. Zheng, J. Chen, Effect of helium and vacancies in a vanadium grain boundary by first-principles, Nucl. Instrum. Methods Phys. Res. Sect. B 352 (2015) 121-124, https://doi.org/10.1016/j.nimb.2015.01.009 (Proceedings of the 12th International Conference on Computer Simulation of Radiation Effects in Solids, Alacant, Spain, 8-13 June, 2014) ISSN 0168-583X. URL <http://www.sciencedirect.com/science/article/pii/S0168583X15000221>.

[19] P. Zhang, T. Zou, J. Zhao, He-he and he-metal interactions in transition metals from first-principles, J. Nucl. Mater. 467 (Part 1) (2015) 465-471, https://doi. org/10.1016/j.jnucmat.2015.09.039, ISSN 0022-3115. URL <http:// www.sciencedirect.com/science/article/pii/S0022311515302282>.

[20] T. Seletskaia, Y. Osetsky, R.E. Stoller, G.M. Stocks, First-principles theory of the energetics of he defects in bcc transition metals, Phys. Rev. B 78 (2008) 134103, https://doi.org/10.1103/PhysRevB.78.134103.

[21] C-C. Fu, J.D. Torre, F. Willaime, J-L. Bocquet, A. Barbu, Multiscale modelling of defect kinetics in irradiated iron, Nat. Mater. 4 (1) (2005) 68-74, https://doi. org/10.1038/nmat1286, ISSN 1476-1122.

[22] G. Kresse, J. Furthmüller, Efficient iterative schemes for $a b$ initio total-energy calculations using a plane-wave basis set, Phys. Rev. B 54 (1996) 1116911186, https://doi.org/10.1103/PhysRevB.54.11169.

[23] G. Kresse, J. Hafner, Ab initio molecular dynamics for liquid metals, Phys. Rev. B 47 (1993) 558-561, https://doi.org/10.1103/PhysRevB.47.558.

[24] J.P. Perdew, K. Burke, M. Ernzerhof, Generalized gradient approximation made simple, Phys. Rev. Lett. 77 (1996) 3865-3868, https://doi.org/10.1103/ PhysRevLett.77.3865.

[25] P.E. Blöchl, Projector augmented-wave method, Phys. Rev. B 50 (1994) 1795317979, https://doi.org/10.1103/PhysRevB.50.17953.

[26] G. Kresse, D. Joubert, From ultrasoft pseudopotentials to the projector augmented-wave method, Phys. Rev. B 59 (1999) 1758-1775, https://doi. org/10.1103/PhysRevB.59.1758.

[27] H.J. Monkhorst, J.D. Pack, Special points for Brillouin-zone integrations, Phys. Rev. B 13 (1976) 5188-5192, https://doi.org/10.1103/PhysRevB.13.5188.

[28] M. Methfessel, A.T. Paxton, High-precision sampling for Brillouin-zone integration in metals, Phys. Rev. B 40 (1989) 3616-3621, https://doi.org/ 10.1103/PhysRevB.40.3616.

[29] G. Henkelman, H. Jónsson, Improved tangent estimate in the nudged elastic band method for finding minimum energy paths and saddle points, J. Chem. Phys. 113 (22) (2000) 9978-9985, https://doi.org/10.1063/1.1323224, URL <http://scitation.aip.org/content/aip/journal/jcp/113/22/10.1063/1.1323224>.

[30] G. Henkelman, B.P. Uberuaga, H. Jónsson, A climbing image nudged elastic band method for finding saddle points and minimum energy paths, J. Chem. Phys. 113 (22) (2000) 9901-9904, https://doi.org/10.1063/1.1329672, URL <http://scitation.aip.org/content/aip/journal/jcp/113/22/10.1063/1.1329672>.
[31] C. Varvenne, F. Bruneval, M-C. Marinica, E. Clouet, Point defect modeling in materials: coupling $a b$ initio and elasticity approaches, Phys. Rev. B 88 (2013) 134102, https://doi.org/10.1103/PhysRevB.88.134102.

[32] C. Kittel, Introduction to Solid State Physics, Wiley, 2004, ISBN 9780471415268. URL <https://books. google.fr/books?id=kym4OgAACAAJ>.

[33] C-C. Fu, F. Willaime, Ab initio study of helium in $\alpha$-Fe: dissolution, migration, and clustering with vacancies, Phys. Rev. B 72 (2005) 064117, https://doi.org/ 10.1103/PhysRevB.72.064117.

[34] K. Maier, M. Peo, B. Saile, H.E. Schaefer, A. Seeger, High-temperature positron annihilation and vacancy formation in refractory metals, Philos. Mag. A 40 (5) (1979) 701-728, https://doi.org/10.1080/01418617908234869.

[35] C. Janot, B. George, P. Delcroix, Point defects in vanadium investigated by Mossbauer spectroscopy and positron annihilation, J. Phys. F: Met. Phys. 12 (1) (1982) 47, URL <http://stacks.iop.org/0305-4608/12/i=1/a=006>.

[36] H. Schultz, J. Takamura, M. Doyama, M. Kiritani, Point defects and defect interactions in metals, in: Proceedings Yamada Conference V, University of Tokyo Press, Tokyo, 1982, p. 183.

[37] H.-E. Schaefer, Investigation of thermal equilibrium vacancies in metals by positron annihilation, Phys. Status Solidi (A) 102 (1) (1987) 47-65, https://doi. org/10.1002/pssa.2211020104.

[38] C.S. Becquart, C. Domain, Ab initio calculations about intrinsic point defects and He in W, Nucl. Instrum. Methods Phys. Res. Sect. B 255 (1)(2007) 23-26, https:// doi.org/10.1016/j.nimb.2006.11.006 (Computer Simulation of Radiation Effects in Solids Proceedings of the Eighth International Conference on Computer Simulation of Radiation Effects in Solids (COSIRES 2006) Computer Simulation of Radiation Effects in Solids). ISSN 0168-583X. URL <http:// www.sciencedirect.com/science/article/pii/S0168583X06010585>.

[39] P.M. Derlet, D. Nguyen-Manh, S.L. Dudarev, Multiscale modeling of crowdion and vacancy defects in body-centered-cubic transition metals, Phys. Rev. B 76 (2007) 054107, https://doi.org/10.1103/PhysRevB.76.054107.

[40] G-Y. Huang, N. Juslin, B.D. Wirth, First-principles study of vacancy, interstitial, noble gas atom interstitial and vacancy clusters in bcc-w, Comput. Mater. Sci. 123 (2016) 121-130, https://doi.org/10.1016/j.commatsci.2016.06.022, ISSN 0927-0256. URL <http://www.sciencedirect.com/science/article/pii/ S0927025616303019>

[41] M. Muzyk, D. Nguyen-Manh, K.J. Kurzydłowski, N.L. Baluc, S.L. Dudarev, Phase stability, point defects, and elastic properties of $\mathrm{W}-\mathrm{V}$ and $\mathrm{W}$-Ta alloys, Phys. Rev. B 84 (2011) 104115, https://doi.org/10.1103/PhysRevB.84.104115.

[42] Y. Oda, A.M. Ito, A. Takayama, H. Nakamura, First-principles study on migration of vacancy in tungsten, Plasma Fusion Res. 9 (2014) 3401117, https://doi.org/10.1585/pfr.9.3401117.

[43] L. Ventelon, F. Willaime, C-C. Fu, M. Heran, I. Ginoux, Ab initio investigation of radiation defects in tungsten: structure of self-interstitials and specificity of di-vacancies compared to other bcc transition metals, J. Nucl. Mater. 425 (1-3) (2012) 16-21, https://doi.org/10.1016/j.jnucmat.2011.08.024 (Microstructure Properties of Irradiated Materials). ISSN 0022-3115. URL <http:// www.sciencedirect.com/science/article/pii/S0022311511008002>.

[44] T. Jourdan, J.-L. Bocquet, F. Soisson, Modeling homogeneous precipitation with an event-based monte carlo method: application to the case of $\mathrm{Fe}-\mathrm{Cu}$, Acta Mater. 58 (9) (2010) 3295-3302, https://doi.org/10.1016/j.actamat.2010.02. 003, ISSN 1359-6454. URL <http://www.sciencedirect.com/science/article/pii/ S1359645410000893>

[45] T. Jourdan, F. Soisson, E. Clouet, A. Barbu, Influence of cluster mobility on cu precipitation in alpha-Fe: a cluster dynamics modeling, Acta Mater. 58 (9)(2010) 3400-3405, https://doi.org/10.1016/j.actamat.2010.02.014, ISSN 1359-6454. URL <http://www.sciencedirect.com/science/article/pii/S135964541000100X>.

[46] T. Jourdan, G. Bencteux, G. Adjanor, Efficient simulation of kinetics of radiation induced defects: a cluster dynamics approach, J. Nucl. Mater. 444 (1-3) (2014) 298-313, https://doi.org/10.1016/j.jnucmat.2013.10.009, ISSN 0022-3115. URL <http://www.sciencedirect.com/science/article/pii/S0022311513011483>.

[47] E. Clouet, A. Barbu, L. Laé, G. Martin, Precipitation kinetics of $\mathrm{Al}_{3} \mathrm{Zr}$ and $\mathrm{Al}_{3} \mathrm{Sc}$ in aluminum alloys modeled with cluster dynamics, Acta Mater. 53 (8) (2005) 2313-2325, https://doi.org/10.1016/j.actamat.2005.01.038, ISSN 1359-6454. URL <http://www.sciencedirect.com/science/article/pii/S1359645405000674>.

[48] J-L. Boutard, S.L. Dudarev, C-C. Fu, F. Willaime, Materials subjected to fas neutron irradiation first principles calculations in iron: structure and mobility of defect clusters and defect complexes for kinetic modelling, C.R. Phys. 9 (3) (2008) 335-342, https://doi.org/10.1016/j.crhy.2007.09.018, ISSN 1631-0705. URL <http://www.sciencedirect.com/science/article/pii/S1631070507002447>.

[49] K. Morishita, R. Sugano, B.D. Wirth, T. Diaz de la Rubia, Thermal stability of helium-vacancy clusters in iron, Nucl. Instrum. Methods Phys. Res. Sect. B 202 (2003) 76-81, https://doi.org/10.1016/S0168-583X(02)01832-3 (6th International Conference on Computer Simulation of Radiation Effects in Solids). ISSN 0168-583X. URL < http://www.sciencedirect.com/science/article/ pii/S0168583X02018323>.

[50] W.D. Wilson, C.L. Bisson, M.I. Baskes, Self-trapping of helium in metals, Phys. Rev. B 24 (1981) 5616-5624, https://doi.org/10.1103/PhysRevB.24.5616.

[51] G.J. Thomas, W.A. Swansiger, M.I. Baskes, Low temperature helium release in nickel, J. Appl. Phys. 50 (11) (1979) 6942-6947, https://doi.org/10.1063 1.325848, URL <http://scitation.aip.org/content/aip/journal/jap/50/11/10 $1063 / 1.325848>$.

[52] G.J. Thomas, R. Bastasz, Direct evidence for spontaneous precipitation of helium in metals, J. Appl. Phys. 52 (10) (1981) 6426-6428, https://doi.org/ 10.1063/1.328590, URL <http://scitation.aip.org/content/aip/journal/jap/52/ 10/10.1063/1.328590>. 
[53] V.S. Subrahmanyam, P.M.G. Nambissan, P. Sen, Thermal evolution of alphainduced defects and helium bubbles in tantalum studied by positron annihilation, J. Phys. Chem. Solids 57 (3) (1996) 319-323, https://doi.org/ 10.1016/0022-3697(95)00284-7, ISSN 0022-3697. URL <http:/ www.sciencedirect.com/science/article/pii/0022369795002847>.

[54] V.S. Subrahmanyam, P. Sen, Helium implanted vanadium studied by the positron annihilation technique, Appl. Radiat. Isot. 46 (10) (1995) 981-985, https://doi.org/10.1016/0969-8043(95)00198-M, ISSN 0969-8043. URL <http://www.sciencedirect.com/science/article/pii/096980439500198M>.

[55] A.V. Fedorov, A. van Veen, A.I. Ryazanov, Nucleation and growth of heliumvacancy clusters in vanadium and vanadium alloys: V-5Ti, V-3Ti-1Si, V-5Ti5Cr, J. Nucl. Mater. 233 (1996) 385-389, https://doi.org/10.1016/S0022-3115 (96)00032-3, ISSN 0022-3115. URL <http://www.sciencedirect.com/science/ article/pii/S0022311596000323>.

[56] R. Li, P. Zhang, C. Zhang, X. Huang, J. Zhao, Vacancy trapping mechanism for multiple helium in monovacancy and small void of vanadium solid, J. Nucl. Mater. 440 (1-3) (2013) 557-561, https://doi.org/10.1016/j.jnucmat. 2013.03.068, ISSN 0022-3115. URL <http://www.sciencedirect.com/science/ article/pii/S0022311513005710>.

[57] A. van Veen, H. Eleveld, M. Clement, Helium impurity interactions in vanadium and niobium, J. Nucl. Mater. 212 (1994) 287-292, https://doi.org/10.1016/ 0022-3115(94)90073-6, ISSN 0022-3115. URL <http://www.sciencedirect. com/science/article/pii/0022311594900736>.

[58] M.B. Lewis, Diffusion of ion implanted helium in vanadium and niobium, J. Nucl. Mater. 152 (2) (1988) 114-122, https://doi.org/10.1016/0022-3115(88) 90317-0, ISSN 0022-3115. URL <http://www.sciencedirect.com/science/ article/pii/0022311588903170>.
[59] R. Vassen, H. Trinkaus, P. Jung, Helium desorption from Fe and V by atomic diffusion and bubble migration, Phys. Rev. B 44 (1991) 4206-4213, https://doi. org/10.1103/PhysRevB.44.4206.

[60] H. Ullmaier, P. Ehrhart, P. Jung, H. Schultz, Atomic defects in metals/Atomare Fehlstellen in Metallen, in: Numerical Data and Functional Relationships in Science and Technology - New Series/Condensed Matter, Springer, Berlin Heidelberg, 1991, ISBN 9783540514350. URL < https://books.google.fr/books? id $=7$ flKAQAAIAAJ>.

[61] K. Faber, J. Schweikhardt, H. Schultz, The intrinsic stage-III annealing in niobium and tantalum following electron irradiation, Scr. Metall. 8 (6) (1974) 713-720, https://doi.org/10.1016/0036-9748(74)90028-3, ISSN 0036-9748. URL <http://www.sciencedirect.com/science/article/pii/0036974874900283>.

[62] H. Trinkaus, B.N. Singh, Helium accumulation in metals during irradiation where do we stand?, J Nucl. Mater. 323 (2-3) (2003) 229-242, https://doi.org/ 10.1016/j.jnucmat.2003.09.001 (Proceedings of the Second IEA Fusion Materials Agreement Workshop on Modeling and Experimental Validation). ISSN 0022-3115. URL <http://www.sciencedirect.com/science/article/pii/ S0022311503004033>.

[63] N. Juslin, B.D. Wirth, Interatomic potentials for simulation of He bubble formation in W, J. Nucl. Mater. 432 (1-3) (2013) 61-66, https://doi.org/ 10.1016/j.jnucmat.2012.07.023, ISSN 00223115. URL <http://linkinghub. elsevier.com/retrieve/pii/S0022311512003820>.

[64] J. Boisse, A. De Backer, C. Domain, C.S. Becquart, Modeling of the self trapping of helium and the trap mutation in tungsten using DFT and empirical potentials based on DFT, J. Mater. Res. 29 (20) (2014) 2374-2386, https://doi. org/10.1557/jmr.2014.258, ISSN 0884-2914, 2044-5326. URL < http://www. journals.cambridge.org/abstract_S0884291414002581>. 